\title{
Implementation of a Monitoring System for Pregnancy by Tele-Ultrasound in an African Context (Burkina Faso)
}

\author{
Seydou Golo BARRO ${ }^{\mathrm{a}, 1}$, Idrissa ZAMPALIGRE ${ }^{\mathrm{b}}$ and Pascal STACCINI ${ }^{\mathrm{c}}$

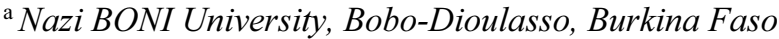 \\ ${ }^{\mathrm{b}}$ Banfora's Sanitary District \\ ${ }^{c}$ RETINES Lab - Cote-d'Azur University, Nice, France
}

\begin{abstract}
Prenatal ultrasound is a radiological examination that allows optimal follow-up of pregnancies. However, its implementation remains limited in poor countries due to a lack of equipment and trained health workers, such as in Burkina Faso. The aim of this work is to set up an ultrasound tele-expertise system. To achieve this objective, we mobilized human, material and IT resources. The design of the tele-expertise application was based on a generic open source software called "MedShakeEHR" that we have adapted to our context. The application runs in a network on a Linux system. It enables ultrasound data exchange and sharing with a remote expert for interpretation using the DICOM protocol. This device thus offers the possibility to pregnant women to carry out their prenatal ultrasound locally. It also allows the constitution of prenatal ultrasound database according security and confidentiality standards.
\end{abstract}

Keywords. Teleexpertise, Ultrasonography, Pregnancy

\section{Introduction}

The realization of obstetric ultrasound in the follow-up of the pregnancy makes it possible to detect in time the risks in both the mother and the fetus. However, in poor countries, this examination is inaccessible to the vast majority of women, and this is mainly due to a lack of equipment and/or trained health workers. In Burkina Faso, especially in rural areas, pregnant women have to travel great distances for a follow-up ultrasound, sometimes not accessible financially. This work is part of this logic of improving the geographic and financial accessibility of obstetric ultrasound. It consists in setting up a technical and organisational system according national and international recommendations [1]:

- design and implementation of a tele-expertise web application from an open source one;

- $\quad$ organization of activities and distribution of tasks.

This device allows pregnant women to perform ultrasounds on the one hand and to interpret ultrasound results remotely. For the experiment of the device, we chose the rural

\footnotetext{
${ }^{1}$ Corresponding Author, Seydou Golo Baroo, University of Nazi BONI, Bobo-Dioulasso, Burkina Faso; E-mail: seydou.golo@gmail.com.
} 
medical center of Niangoloko, Burkina Faso, as a pilot site for the deployment of the device in order to test his different functionalities.

\section{Method}

Analysis of the existing system shows this problem: pregnant women in rural areas don't have the opportunity to perform ultrasound on site due to lack of equipment and trained health workers. During the prenatal consultation, ultrasound is then prescribed as an external examination to be performed in a specialized medical center.

These centers are mostly located in other locations. For our example at Niangoloko, the nearest specialized medical center is the Banfora Regional Hospital Center located $45 \mathrm{~km}$ away.

The system set up takes account of the existing situation and includes technical IT and organisational component. For the technical and computer aspects, we chose opensource software that has proven to be effective. These applications have been installed on the Linux operating system (Ubuntu 18.04.5 LTS). It concerns:

- MedShakeEHR, free medical software that we used for the design of the teleexpertise application [2].

- Orthanc, standalone DICOM server, for ultrasound data management [3].

We have made changes to the software to adapt it to our project. All actors have been trained to use it. On the organisational side, the midwife at the rural medical center is responsible for handling the ultrasound system for the examination of the woman and forwarding the ultrasound data to a physician expert for interpretation. This expert is then responsible for forwarding to the staff of the rural health center a written report, attached to the ultrasound images that are given to the patient by appointment.

\section{Results}

\subsection{Application design and implementation}

We used MedShakeEHR-base and MedShakeEHR-modGynObs modules of the MedShakeEHR software, in which we made modifications to adapt them to our project.

The following changes were made:

- implementation of a unique patient identification algorithm from the person identification profile (strict traits, extended traits, complementary traits) [4,5];

- $\quad$ integration of a database of localities in the country (village, sector,...);

- integration of a reference system for the country's professions;

- integration of a ICD-10 database for pathological diagnosis.

Fields on the base form that didn't meet our needs have been removed. The finalized application was installed on the CFRTM (Medical Technology Research and Training Center) server with remote access. All actors (assistants, midwives, specialists and supervisors) were trained in the use of tele-expertise application by their level of intervention. Midwives have received essential training, during six months from 
specialist (gynecologist-obstetrician doctor), to perform an obstetric ultrasound: collection of ultrasound measurements and images, transmission of data.

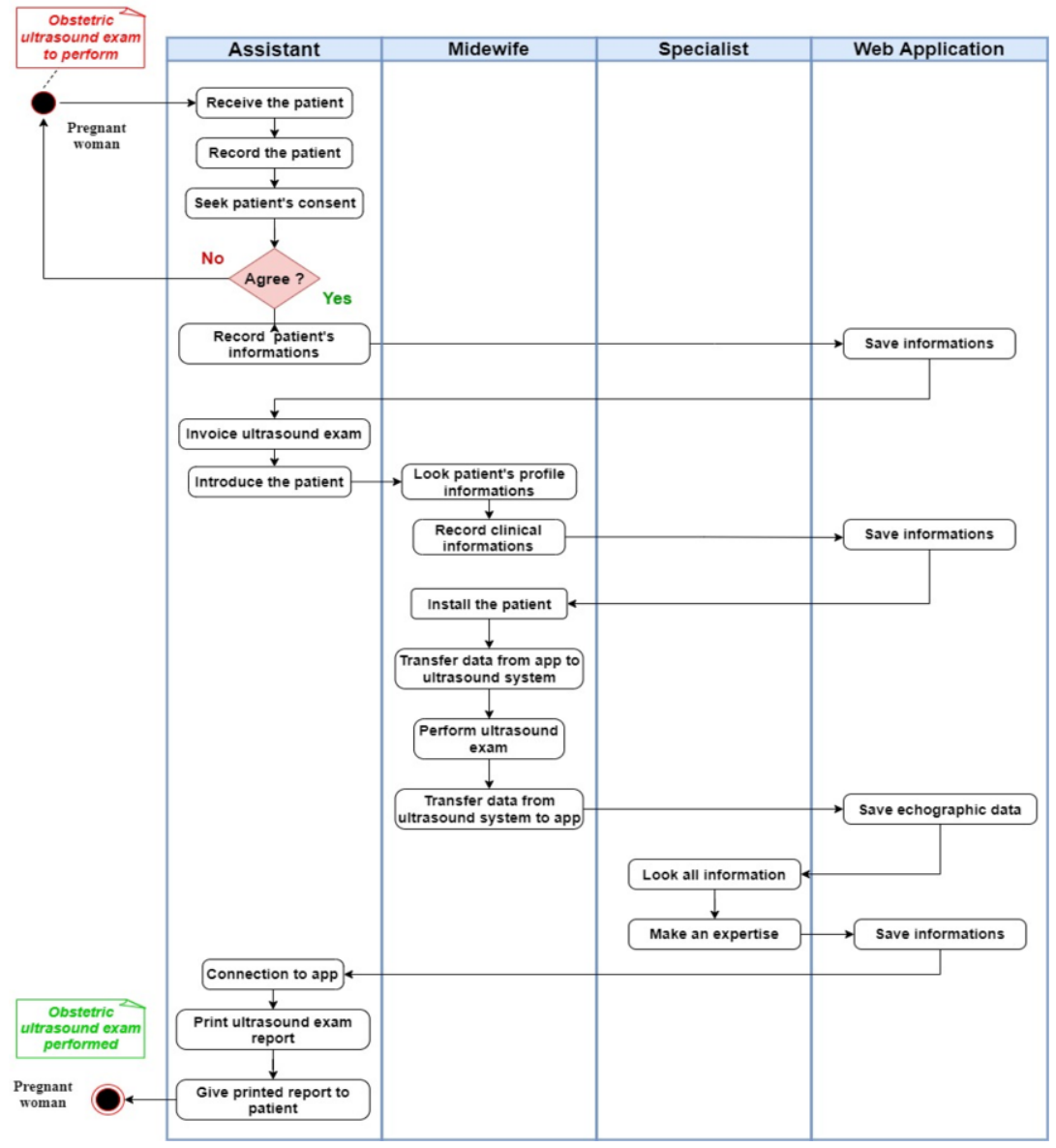

Figure 1. Device operation

\subsection{Device operation}

Ultrasound activities are carried out on three working days during a week: monday, wednesday and friday.

- ultrasound is performed in the morning from 08:00 to 12:00. After 12:00, a nominative list with the patient ID identifiers of the day are sent by email to the gynecologist-obstetrician doctor no later than 14:00.

- the gynecologist-obstetrician doctor connects to the application, consults the various records of the day and delivers his expertise according to his availability within 24 hours.

- The report of the prenatal ultrasound is therefore available; it will be printed and given to the patient on an appointment fixed beforehand in agreement with her within 48 hours.

At the decision of the steering committee, ultrasound examinations are invoiced at 5.000 CFA franc (\$9) against 6000 CFA franc (\$11) applied in public health 
establishments excluding transport costs. The operation of the device, from patient reception to ultrasound interpretation is described by the following diagram.

\subsection{Patient data security and confidentiality}

Since we worked with health information, which is therefore highly confidential, it was crucial to ensure the security of health data as governed by Article 15 of Law 0102004/AN on the protection of personal data [6].

Steps have been taken to ensure this confidentiality:

- a single administrator;

- $\quad$ encryption of the data storage hard drive on the server;

- an automatic backup system on external media;

- user authentication;

- a limitation of users' access rights according to their profile;

- a firewall to protect our server from remote attacks.

\section{Discussion and conclusions}

One of the major reasons for the low completion of ultrasound for pregnancy follow-up is the lack of availability of this examination in rural areas, which is essential for the smooth running of pregnancy. The main advantage of the ultrasound tele-expertise system we have set up is the accessibility of this examination, which is a guarantee of the motivation of rural populations. It is also a source of motivation for the staff of rural health centres who feel more involved in monitoring pregnant women.

Beyond this work objective, the scheme will eventually allow the creation of a database for monitoring pregnant women, which is necessary for the production of reliable statistics and research. The unique identification system implemented could open avenues for its extension to the HIS of our country.

\section{References}

[1] Conférence Nationale d'Echographie Obstétricale et Fœtale. L'échographie de dépistage prénatal. 2016. Available from: (http:/www.cngof.fr/component/rsfiles/telechargement-fichier/fichiers?path= Clinique\%252Finfo\%2Bpatientes\%252Fcngof_info_17-echographie_grossesse_pdf\&Itemid=756).

[2] Logiciel cabinet médical. MedShakeEHR : logiciel modulaire, universel, open source pour les praticiens santé (et son 1er module pour la gynécologie obstétrique). Available from: https://www.medshake.net/ blog/a199/medshakeehr-logiciel-modulaire-universel-open-source-pour-les-praticiens-sante-et-son-1ermodule-pour-la-gynecologie-obstetrique/. Date accesed: 25/05/2021.

[3] Orthanc-DICOM Server. About. https://www.orthanc-server.com/static.php?page=about. Date accesed: 25/05/2021

[4] ANAP. Identification du patient. Access date: 29/05/2021. https://www.anap.fr/ressources/publications/ detail/actualites/identification-du-patient/.

[5] Programme Identifiant National calculé (INS-C) de Santé, Algorithme de calcul. ASIP Santé / PRAS ed. Paris: ASIP Santé, 2009;5-9. Available from: (https://esante.gouv.fr/securite/identifiant-national-desante/ins-c).

[6] CIL. Législation nationale. http://www.cil.bf/index.php/legislation/legislation-nationale. Date accesed: $31 / 05 / 2019$. 